\title{
Isotropic to Anisotropic Transition Observed in Si Nanoparticles Lithiation by in situ TEM
}

\author{
Jinsong $\mathrm{Wu}^{1,2}$, Langli Luo ${ }^{1}$, Jiayan Luo $^{2}$, Jiaxing Huang ${ }^{2}$ and Vinayak P. Dravid ${ }^{1,2}$ \\ 1. NUANCE Center, Northwestern University, Evanston, IL 60208, United States \\ 2. Department of Materials Science and Engineering, Northwestern University, Evanston, IL 60208, \\ United States
}

$\mathrm{Si}$ is a promising candidate anode material for the next generation Li-ion battery because of its high capacity. However, Si inherently suffers from its large volume change $(\sim 300 \%)$ when alloyed with $\mathrm{Li}$ during the typical charge/discharge cycles, which degrades battery performance and causes potential safety issues. Understanding of microstructural evolutions of $\mathrm{Si}$ in the lithiation/delithiation cycles is helpful to design better electrodes. In-situ TEM has been recently applied to study dynamically microstructural evolution of pure $\mathrm{Si}$ in the electrochemical lithiation/delithiation reaction [1-6]. Crystalline Si undergoes a two-phase process upon the first lithiation, with an interface between amorphous $\mathrm{Li}_{\mathrm{x}} \mathrm{Si}$ phase that is formed during the alloying reaction of $\mathrm{Si}$ and $\mathrm{Li}$, and the remainder (unreacted) crystalline Si in the center. The lithiation of amorphous Si has similar two-phase process, where the interface is defined between Li-poor core and Li-rich shell regions. The first lithiation of crystalline $\mathrm{Si}$ is anisotropic and dominated by the alloying reaction at the interface [2-4]. The first lithiation of amorphous Si nanoparticle is, however, isotropic [1]. The critical threshold of generating fracture for amorphous Si nanoparticles $(870 \mathrm{~nm})$ is much higher than that of the crystalline ones $(150$ $\mathrm{nm})[1]$.

We studied the lithiation of crystalline $\mathrm{Si}$ nanoparticles encapsulated by the graphene sheets (Si/Graphene) by in-situ TEM [5]. Unexpectedly, in the first lithiation process, crystalline Si nanoparticles undergo an isotropic to anisotropic transition, which is not observed in pure crystalline and amorphous $\mathrm{Si}$ nanoparticles. As shown in fig. 1a-f, the reaction interface between the core (crystalline $\mathrm{Si}$ ) and shell (amorphous $\mathrm{Li}_{\mathrm{x}} \mathrm{Si}$ ) changes from isotropic to anisotropic as the lithiation proceeds. Such a surprising phenomenon arises from the uniformly distributed localized potential around the Si nanoparticles due to the highly conductive graphene sheets, as schematically shown in fig.1g. The phase transformation of Si in the in-situ lithiation/delithiation reactions has also been studied by electron diffraction. Upon first lithiation, a core-shell structure with crystalline $\mathrm{Si}$ core and amorphous $\mathrm{Li}_{\mathrm{X}} \mathrm{Si}$ shell is formed. As shown in Fig.2a, the diffraction pattern taken from a partially lithiated $\mathrm{Si} /$ Graphene nanoparticles consists of diffraction spots from crystalline $\mathrm{Si}$ (core) and diffuse diffraction rings from amorphous $\mathrm{Li}_{\mathrm{x}} \mathrm{Si}$ shell. At the late stage of the first lithiation, a phase transformation from amorphous $\mathrm{Li}_{\mathrm{x}} \mathrm{Si}$ to nano-sized crystal $\mathrm{Li}_{15} \mathrm{Si}_{4}$ can be identified as shown in fig.2b-c.

Unlike pure crystalline $\mathrm{Si}$ nanoparticle, the lithiation of $\mathrm{Si} / \mathrm{Graphene}$ nanoparticle is isotropic at the beginning. This could lead to a more uniformly distributed stress in the nanoparticle thus improvement in cycling stability, similar to that with amorphous Si nanoparticles. These findings offer guidance to designing of improved nanostructured $\mathrm{Si}$ materials as fast and large-capacity anode materials for lithium ion batteries.

\section{References:}

[1] M.T. McDowell, et al. Nano Letters 13, (2013), p.758.

[2] M.T. McDowell, et al. Advanced Materials 24, (2012), p. 6034. 
[3] X.H. Liu, et al. Nano Letters 11, (2011), p. 3312.

[4] X.H. Liu, et al. ACS Nano 6, (2012), p.1522.

[5] L. Luo, et al. Scientific Reports 4, (2014), article number 3863.
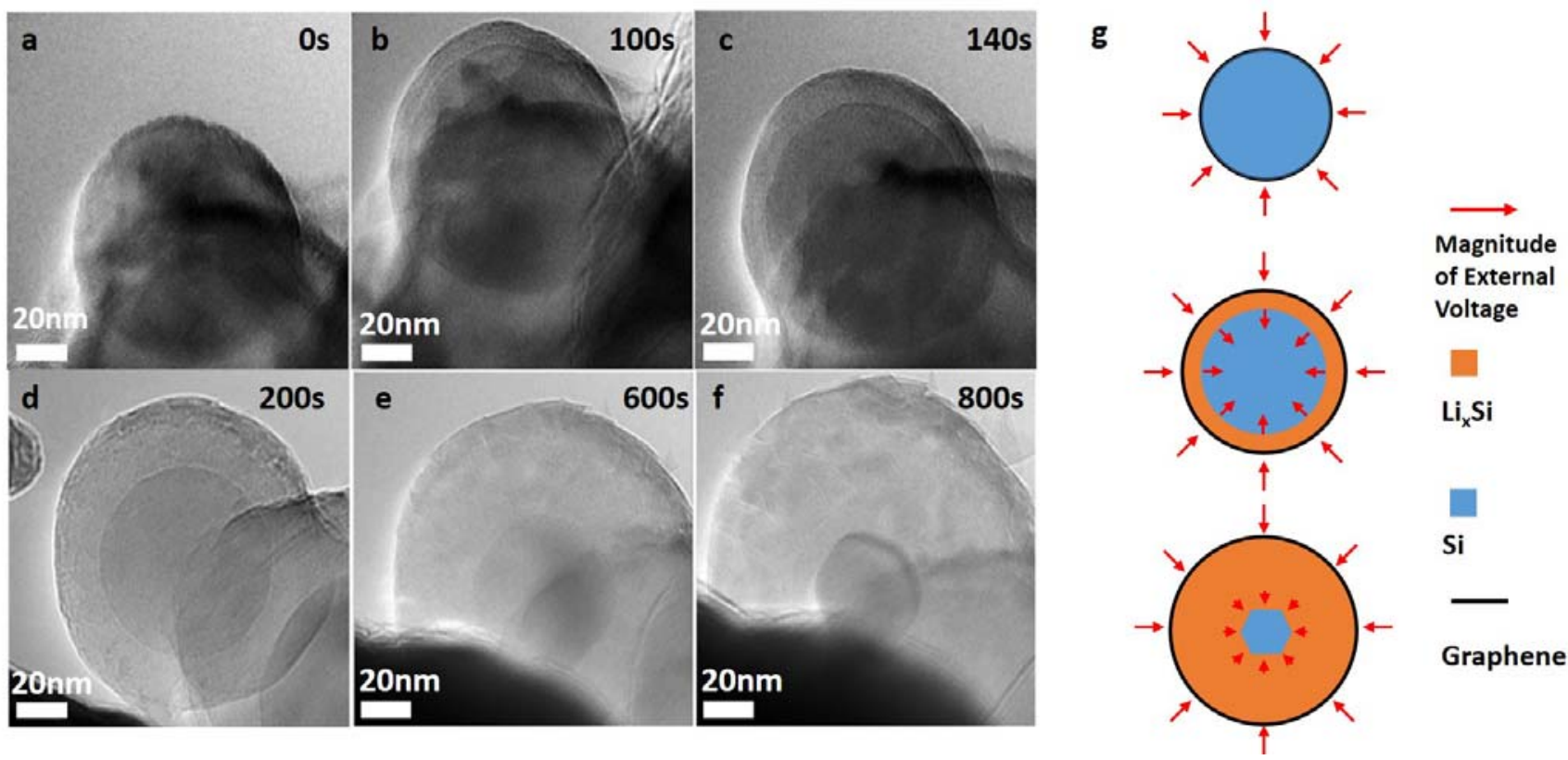

Figure 1. The first lithiation of a single graphene sheet encapsulated Si nanoparticles with lithiation time of (a) $0 \mathrm{~s}$, (b) 100s, (c) 140s, (d) 200s, (e) 600s and (f) 800s; (g) schematic of isotropic to anisotropic lithiation of crystalline Si nanoparticle. The magnitude of localized external voltage/work applied at the $\mathrm{Si} / \mathrm{Li}_{\mathrm{x}} \mathrm{Si}$ interface (reaction front) decreases along with the lithiation as indicated by read arrows.

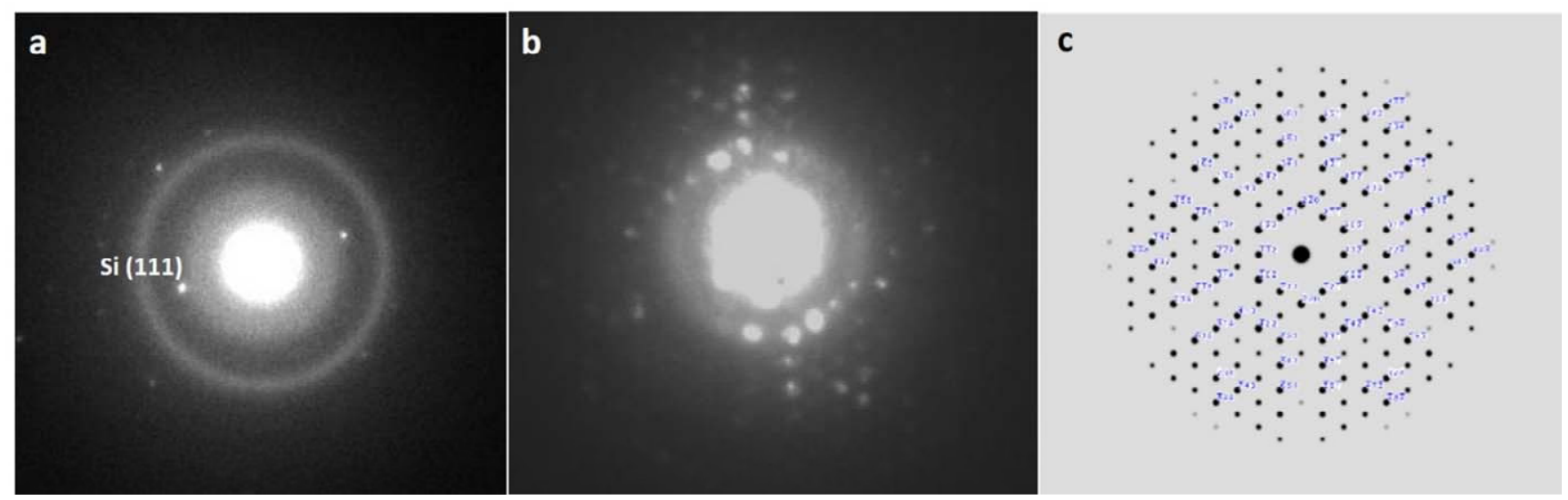

Figure 2. Phase transformation in the lithiation process. (a) Selected-area electron diffraction of a partial lithiated $\mathrm{Si} /$ graphene nanoparticles, showing amorphous ring of $\mathrm{Li}_{\mathrm{x}} \mathrm{Si}$ and diffraction spots of the $\mathrm{Si}$ core. (b) A nano-beam electron diffraction collected from a area in the fully lithiated Si/Graphene nanoparticle, which can be indexed as [111] zone axis of $\mathrm{Li}_{15} \mathrm{Si}_{4}$ crystal. (c) Simulated SAED pattern of $\mathrm{Li}_{15} \mathrm{Si}_{4}$ crystal along the [111] axis. 